$2-1-2012$

\title{
A novel practical control approach for rate independent hysteretic systems
}

Frank J. Goforth

Georgia Southern University, fgoforth@georgiasouthern.edu

Qing Zhengb

Gannon University, zheng003@gannon.edu

Zhiqiang Gao

Cleveland State University, Z.GAO@csuohio.edu

Follow this and additional works at: https://engagedscholarship.csuohio.edu/enece_facpub

How does access to this work benefit you? Let us know!

\section{Publisher's Statement}

NOTICE: this is the author's version of a work that was accepted for publication in ISA

Transactions. Changes resulting from the publishing process, such as peer review, editing, corrections, structural formatting, and other quality control mechanisms may not be reflected in this document. Changes may have been made to this work since it was submitted for publication. A definitive version was subsequently published in ISA Transactions, 51, 3, (02-01-2012); 10.1016/j.isatra.2012.01.006

\section{Repository Citation}

Goforth, Frank J.; Zhengb, Qing; and Gao, Zhiqiang, "A novel practical control approach for rate independent hysteretic systems" (2012). Electrical Engineering \& Computer Science Faculty Publications. 255.

https://engagedscholarship.csuohio.edu/enece_facpub/255

This Article is brought to you for free and open access by the Electrical Engineering \& Computer Science Department at EngagedScholarship@CSU. It has been accepted for inclusion in Electrical Engineering \& Computer Science Faculty Publications by an authorized administrator of EngagedScholarship@CSU. For more information, please contact library.es@csuohio.edu. 


\title{
A novel practical control approach for rate independent hysteretic systems
}

\author{
Frank J. Goforth ${ }^{\mathrm{a}, 1}$, Qing Zheng ${ }^{\mathrm{b}, *}$, Zhiqiang Gao ${ }^{\mathrm{c}, 2}$ \\ ${ }^{a}$ Department of Mechanical \& Electrical Engineering, Georgia Southern University, Statesboro, GA 30460 USA \\ ${ }^{b}$ Department of Electrical and Computer Engineering, Gannon University, Erie, PA 16541, USA \\ ${ }^{\mathrm{C}}$ Department of Electrical and Computer Engineering, Cleveland State University, Cleveland, $\mathrm{OH} 44115$, USA
}

\section{Introduction}

Various hysteresis models have been investigated in research [1-15]. Hysteresis compensation has generally followed three alternatives: feedforward compensation, classic "prefilter" configuration, and the application of an inverse of the hysteresis nonlinearity in series with the process. However, all the above alternatives require a model of the hysteresis or the success depends on how accurate the model of the hysteresis is. Unfortunately, modeling of the hysteresis is very challenging. The model based compensation requires long compute cycles to resolve and must be calibrated and tuned regularly to account for nonlinear drift and offsets.

Inspired by recent contributions regarding the hysteretic system control [8-15], the active disturbance rejection control (ADRC) is proposed as an alternative paradigm to address the hysteretic problems in this paper $[16,17]$. The main difference in the design concept pertains to the question of how much model information is needed. Recognizing the vulnerability of the reliance on accurate mathematical model, there has been a gradual

\footnotetext{
* Corresponding author. Tel.: +1 814871 5617; fax: +1 8148717616 . E-mail addresses: fgoforth@georgiasouthern.edu (F.J. Goforth), zheng003@gannon.edu (Q.Zheng).z.gao@csuohio.edu (Z. Gao).

1 Tel.: +1 9124787583 ; fax: +1912478 1455..

2 Tel.: +1 216687 3528; fax: +1 2166875405 .
}

recognition over the years that active disturbance estimation is a viable alternative to an accurate plant model. That is, if the disturbance, representing the discrepancy between the plant and its model, is estimated in real time, then the plant-model mismatch can be effectively compensated for, making the model based design tolerant of a large amount of uncertainties. The focal point is how external disturbance and unknown dynamics can be estimated.

Several classes of approach have been proposed to deal with systems with unknown dynamics, including the unknown input observer (UIO) $[18,19]$, the disturbance observer (DOB) $[20,21]$, the perturbation observer (POB) [22], and the extended state observer (ESO) $[16,17,23]$. All the above estimators prove to be effective practical solutions. However, how fast and in what range the disturbance and unknown dynamics can be estimated are not obvious.

The purpose of this paper is to provide a practical solution based on the ADRC technology to hysteretic systems and show analytically how ADRC achieves excellent performance, even when the plant of hysteric systems is unknown, nonlinear and timevarying. The convergence and the bounds of the estimation and tracking errors are presented.

This paper is organized as follows. A general class of hysteretic systems is described in Section 2. The ADRC approach and its stability analysis are presented in Section 3. Simulation tests on a typical hysteric system are shown in Section 4. The paper ends with concluding remarks in Section 5. 


\section{Hysteretic systems}

A general class of hysteretic systems is described in this section. The time scales for nonlinearities other than hysteresis, such as thermal drift and voltage creep, are normally orders of magnitude slower than that of hysteresis and are herein ignored. The choice is made, in the spirit of singular perturbation theory but as assumption, to address hysteresis decoupled from slower nonlinearities.

\subsection{Hysteresis nonlinearity}

Hysteretic response may be at times $20 \%$ or more nonlinear. The issues caused by this nonlinearity are exacerbated in many applications where the normal operating environment requires transiting the hysteretic region constantly and rapidly. A consequence of this hysteresis is the limited bandwidth of operation, achieving desired performance only at reduced operating speeds.

\subsection{Typical hysteretic systems}

In the various investigated hysteresis models, the physical responses of hysteretic processes are unique to each, and differ in symmetry, saturation, and swept area, but are generally similar in their anhysteretic small signal response and large signal saturation. Various hysteresis operators have strengths and weaknesses in their capabilities to accurately represent these differences, and this is critical when one is dependent on that model for control. However, the ability to represent the rate independent memory of hysteresis in the actuator model is necessary to best demonstrate the strength of any controller. The proposed controller does not depend on the model of hysteresis. Its stability and ability to reject disturbance depend on the bounded nature of the disturbance.

Let us consider a general class of hysteretic systems. The full motion equation can be reformulated as:

$x^{(n)}(t)=f\left(x^{(n-1)}(t), x^{(n-2)}(t), \ldots, x(t), d_{t}(t)\right)+b u(t)$

where $d_{t}$ refers to the total disturbance including linear and nonlinear disturbances with unknown characteristics. The various linear elastic terms and nonlinear contributions from hysteresis, creep, drift, etc. are combined as part of the generalized nonlinear function $f(\cdot)$, simply denoted as $f$.

This reformulation of the motion control problem in (1) leads us to a new solution shown in the next section. The key idea is the recognition that the control design does not have to necessarily depend on the exact mathematical expression of $f$, so long as its value can be estimated in real time from the input and output data, i.e. $x(t)$ and $u(t)$. If a good estimate of $f$ can be obtained from an observer, say the ESO, then by canceling $f$, using its estimate, the control system is reduced to a simple repeating real pole controller design for the nominal $n$-dimension integral plant. Such design strategy of actively rejecting the unknown based on its real time estimation is known as ADRC, as described below.

\section{Active disturbance rejection control}

The prevailing technique for hysteresis compensation has been model inversion, which achieves resolution and precision, but must be calibrated for each application and is computationally intense to operate in real time. This research proposes an entirely different hysteresis compensation: treat the nonlinear hysteresis as a common disturbance to the desired linear response, and actively reject that disturbance to present a compensated system to the controller which responds linearly, indeed as a simple $n$-dimension integrator. An ESO is implemented wherein the effect of the hysteresis nonlinearity is treated as disturbance and canceled. The hysteresis will be compensated such that the resulting equivalent $n$-dimension integrator presented to the position controller may then be easily managed by a proportional plus derivative (PD) control. The explanation of the ADRC/ESO contained in this section, in the interest of clarity, will be described for a common second order system. The ESO, ADRC, and their convergence analyses for a general $n$th order system are given in [24].

\subsection{Extended state observer design}

Let $\xi_{1}=x, \xi_{2}=\dot{x}, \xi_{3}=f$ and $\xi=\left[\begin{array}{lll}\xi_{1} & \xi_{2} & \xi_{3}\end{array}\right]^{T}$. Assuming $f$ is differentiable, the state space form of $(1)$ is

$\left\{\begin{array}{l}\dot{\xi}=A \xi+B u+E h \\ x=C \xi\end{array}\right.$

where $A=\left[\begin{array}{lll}0 & 1 & 0 \\ 0 & 0 & 1 \\ 0 & 0 & 0\end{array}\right], B=\left[\begin{array}{l}0 \\ b \\ 0\end{array}\right], C=\left[\begin{array}{lll}1 & 0 & 0\end{array}\right], E=\left[\begin{array}{l}0 \\ 0 \\ 1\end{array}\right]$, $\xi_{3}=f$ is the augmented state, and $h=\dot{f}$. A continuous ESO for (2) is designed as

$\left\{\begin{array}{l}\dot{\hat{\xi}}=A \hat{\xi}+B u+L(x-\hat{x}) \\ \hat{x}=C \hat{\xi}\end{array}\right.$

where $L=\left[\begin{array}{lll}l_{1} & l_{2} & l_{3}\end{array}\right]^{T}$ is the observer gain vector. The observer gains are chosen such that the characteristic polynomial $s^{3}+l_{1} s^{2}+$ $l_{2} s+l_{3}$ is Hurwitz. For tuning simplicity, all the observer poles are placed at $-\omega_{0}$. It results in the characteristic polynomial of (3) to be

$\lambda_{0}(s)=s^{3}+l_{1} s^{2}+l_{2} s+l_{3}=\left(s+\omega_{0}\right)^{3}$

where $\omega_{0}$ is the observer bandwidth and $L=\left[\begin{array}{lll}3 \omega_{0} & 3 \omega_{0}^{2} & \omega_{0}^{3}\end{array}\right]^{T}$.

Generally, the larger the observer bandwidth, the more accurate the estimation. However, a large observer bandwidth will increase noise sensitivity. Therefore a proper observer bandwidth should be selected in a compromise between the estimation performance and the noise tolerance.

\subsection{Control algorithm}

Once the observer is designed and well tuned, its outputs will track $\xi_{1}, \xi_{2}$, and $\xi_{3}$ respectively. By canceling the effect of $f$ using $\hat{\xi}_{3}$, ADRC actively compensates for $f$ in real time. The ADRC control law is given by

$u_{d}=\frac{k_{1}\left(r-\hat{\xi}_{1}\right)+k_{2}\left(\dot{r}-\hat{\xi}_{2}\right)-\hat{\xi}_{3}+\ddot{r}}{b}$

where $r$ is the desired trajectory, $k_{1}$ and $k_{2}$ are the controller gain parameters selected to make $s^{2}+k_{2} s+k_{1}$ Hurwitz. For simplicity, let $k_{1}=\omega_{c}^{2}, k_{2}=2 \omega_{c}$, where $\omega_{c}$ is the controller bandwidth. The closed-loop system for the system (1) becomes

$\ddot{x}=\left(f-\hat{\xi}_{3}\right)+k_{1}\left(r-\hat{\xi}_{1}\right)+k_{2}\left(\dot{r}-\hat{\xi}_{2}\right)+\ddot{r}$.

Note that with a well-designed ESO, the first term in the right hand side (RHS) of (6) is negligible and the rest of the terms in the RHS of (6) constitute a PD controller with a feedforward gain.

In practice, the controller bandwidth $\omega_{c}$ is tuned based on how fast we want the output to track the set point. A large controller bandwidth generally increases the response speed but it may push the system to its limit, leading to oscillations or even instability. Thus the controller bandwidth should be adjusted based on the 
competing requirements of performance and stability margin, together with noise sensitivity. In addition, a large controller bandwidth usually increases the magnitude and rate of change in control signal, and therefore the operation cost.

The convergence for the estimation error of the ESO and the closed-loop tracking error of ADRC is shown below.

\subsection{Stability}

(1) Convergence of the ESO

Let $\tilde{\xi}_{i}(t)=\xi_{i}(t)-\hat{\xi}_{i}(t), i=1,2$, 3. From (2) and (3), the observer estimation error dynamics can be shown as

$\dot{\tilde{\xi}}_{1}=\tilde{\xi}_{2}-l_{1} \tilde{\xi}_{1}$

$\dot{\tilde{\xi}}_{2}=\tilde{\xi}_{3}-l_{2} \tilde{\xi}_{1}$

$\dot{\tilde{\xi}}_{3}=h-l_{3} \tilde{\xi}_{1}$.

Now let us scale the observer estimation error $\tilde{\xi}_{i}(t)$ by $\omega_{o}^{i-1}$, i.e., let $\varepsilon_{i}(t)=\frac{\tilde{\xi}_{i}(t)}{\omega_{0}^{i-1}}, i=1,2,3$. Then (7) can be rewritten as

$\dot{\varepsilon}=\omega_{0} A_{\varepsilon} \varepsilon+B_{\varepsilon} \frac{h\left(\xi, d_{t}\right)}{\omega_{o}^{2}}$

where $A_{\varepsilon}=\left[\begin{array}{lll}-3 & 1 & 0 \\ -3 & 0 & 1 \\ -1 & 0 & 0\end{array}\right], B_{\varepsilon}=\left[\begin{array}{l}0 \\ 0 \\ 1\end{array}\right]$,and $d_{t}$ refers to the total disturbance.

Theorem 1. Assuming $h\left(\xi, d_{t}\right)$ is bounded, then there exist a constant $\sigma_{i}>0$ and a finite time $T_{1}>0$ such that $\left|\tilde{\xi}_{i}(t)\right| \leq \sigma_{i}$, $i=1,2,3, \forall t \geq T_{1}>0$ and $\omega_{0}>0$. Furthermore, $\sigma_{i}=O\left(\frac{1}{\omega_{0}^{k}}\right)$, for some positive integer $k$.

Proof. Solving (8), we can obtain

$\varepsilon(t)=e^{\omega_{0} A_{\varepsilon} t} \varepsilon(0)+\int_{0}^{t} e^{\omega_{0} A_{\varepsilon}(t-\tau)} B_{\varepsilon} \frac{h\left(\xi(\tau), d_{t}\right)}{\omega_{0}^{2}} d \tau$.

Let

$p(t)=\int_{0}^{t} e^{\omega_{0} A_{\varepsilon}(t-\tau)} B_{\varepsilon} \frac{h\left(\xi(\tau), d_{t}\right)}{\omega_{o}^{2}} d \tau$.

Since $h\left(\xi(\tau), d_{t}\right)$ is bounded, that is, $\left|h\left(\xi(\tau), d_{t}\right)\right| \leq \delta$, where $\delta$ is a positive constant, it follows that

$\left|p_{i}(t)\right| \leq \frac{\delta}{\omega_{0}^{3}}\left[\left|\left(A_{\varepsilon}^{-1} B_{\varepsilon}\right)_{i}\right|+\left|\left(A_{\varepsilon}^{-1} e^{\omega_{0} A_{\varepsilon} t} B_{\varepsilon}\right)_{i}\right|\right]$

for $i=1,2$, 3. Since $A_{\varepsilon}^{-1}=\left[\begin{array}{lll}0 & 0 & -1 \\ 1 & 0 & -3 \\ 0 & 1 & -3\end{array}\right]$, One has

$\left|\left(A_{\varepsilon}^{-1} B\right)_{i}\right|=\left\{\begin{array}{l}\left.1\right|_{i=1} \\ \left.3\right|_{i=2,3}\end{array}\right.$

Since $A_{\varepsilon}$ is Hurwitz, there exists a finite time $T_{1}>0$ such that

$\left|\left[e^{\omega_{0} A_{\varepsilon} t}\right]_{i j}\right| \leq \frac{1}{\omega_{0}^{3}}$

for all $t \geq T_{1}, i, j=1,2,3$. Hence

$$
\left|\left[e^{\omega_{0} A_{\varepsilon} t} B\right]_{i}\right| \leq \frac{1}{\omega_{0}^{3}}
$$

for all $t \geq T_{1}, i=1,2,3$. Note that $T_{1}$ depends on $\omega_{0} A_{\varepsilon}$.
Let $A_{\varepsilon}^{-1}=\left[\begin{array}{lll}s_{11} & s_{12} & s_{13} \\ s_{21} & s_{22} & s_{23} \\ s_{31} & s_{32} & s_{33}\end{array}\right]$ and $e^{\omega_{0} A_{\varepsilon} t}=\left[\begin{array}{lll}d_{11} & d_{12} & d_{13} \\ d_{21} & d_{22} & d_{23} \\ d_{31} & d_{32} & d_{33}\end{array}\right]$. One has $\left|\left(A_{\varepsilon}^{-1} e^{\omega_{0} A_{\varepsilon} t} B_{\varepsilon}\right)_{i}\right|=\left|s_{i 1} d_{13}+s_{i 2} d_{23}+s_{i 3} d_{33}\right|$

$$
\leq\left\{\begin{array}{l}
\frac{1}{\omega_{o}^{3}} \\
\left.\frac{4}{\omega_{o}^{3}}\right|_{i=2,3}
\end{array}\right.
$$

for all $t \geq T_{1}$. From (11), (12) and (15), we obtain

$\left|p_{i}(t)\right| \leq \frac{3 \delta}{\omega_{0}^{3}}+\frac{4 \delta}{\omega_{o}^{6}}$

for all $t \geq T_{1}, i=1,2,3$. Let $\varepsilon_{\text {sum }}(0)=\left|\varepsilon_{1}(0)\right|+\left|\varepsilon_{2}(0)\right|+\left|\varepsilon_{3}(0)\right|$. It follows that

$\left|\left[e^{\omega_{0} A_{\varepsilon} t} \varepsilon(0)\right]_{i}\right| \leq \frac{\varepsilon_{\text {sum }}(0)}{\omega_{0}^{3}}$

for all $t \geq T_{1}, i=1,2,3$. From (9), one has

$\left|\varepsilon_{i}(t)\right| \leq\left|\left[e^{\omega_{0} A_{\varepsilon} t} \varepsilon(0)\right]_{i}\right|+\left|p_{i}(t)\right|$.

Let $\tilde{\xi}_{\text {sum }}(0)=\left|\tilde{\xi}_{1}(0)\right|+\left|\tilde{\xi}_{2}(0)\right|+\left|\tilde{\xi}_{3}(0)\right|$. According to $\varepsilon_{i}(t)=$ $\frac{\tilde{\xi}_{i}(t)}{\omega_{0}^{i-1}}$ and (16)-(18), we have

$$
\begin{aligned}
\left|\tilde{\xi}_{i}(t)\right| & \leq\left|\frac{\tilde{\xi}_{\text {sum }}(0)}{\omega_{o}^{3}}\right|+\frac{3 \delta}{\omega_{0}^{4-i}}+\frac{4 \delta}{\omega_{o}^{7-i}} \\
& =\sigma_{i}
\end{aligned}
$$

for all $t \geq T_{1}, i=1,2,3$.

It is shown above that in the absence of the plant model, the estimation error of the ESO (3) is bounded and its upper bound monotonously decreases with the observer bandwidth. The meaning of the assumption that $h\left(\xi, d_{t}\right)$ is bounded is: there is a limit to the rate of change in the physical world, or no change is instantaneous. When $f$ is a composite variable that changes very rapidly, the magnitude of $\dot{f}$ can be quite large, though bounded. In this case, the observer bandwidth needs to be sufficiently large for an accurate estimate of $f$.

The convergence of ADRC, where ESO is employed, is analyzed next.

(2) Convergence of the ADRC

Let $\left[r_{1}, r_{2}, r_{3}\right]^{T}=[r, \dot{r}, \ddot{r}]^{T}$ and $e_{i}(t)=r_{i}(t)-\xi_{i}(t), i=1,2$.

Theorem 2. Assuming that $h$ is bounded, there exist a constant $\rho_{i}>$ 0 and a finite time $T_{3}>0$ such that $\left|e_{i}(t)\right| \leq \rho_{i}, i=1,2, \forall t \geq$ $T_{3}>0, \omega_{0}>0$ and $\omega_{c}>0$.

Furthermore, $\rho_{i}=O\left(\frac{1}{\omega_{c}^{q}}\right)$ for some positive integer $q$.

Proof. From (5), one has

$u=\frac{k_{1}\left(e_{1}+\tilde{\xi}_{1}\right)+k_{2}\left(e_{2}+\tilde{\xi}_{2}\right)-\left(\xi_{3}-\tilde{\xi}_{3}\right)+r_{3}}{b}$.

It follows that

$\dot{e}_{1}=\dot{r}_{1}-\dot{\xi}_{1}=r_{2}-\xi_{2}=e_{2}$

$\dot{e}_{2}=-k_{1}\left(e_{1}+\tilde{\xi}_{1}\right)-k_{2}\left(e_{2}+\tilde{\xi}_{2}\right)-\tilde{\xi}_{3}$.

Let $e(t)=\left[e_{1}(t), e_{2}(t)\right]^{T}, \tilde{\xi}(t)=\left[\tilde{\xi}_{1}(t), \tilde{\xi}_{2}(t), \tilde{\xi}_{3}(t)\right]^{T}$, then

$\dot{e}(t)=A_{e} e(t)+A_{\tilde{\xi}} \tilde{\xi}(t)$ 
where $A_{e}=\left[\begin{array}{cc}0 & 1 \\ -k_{1} & -k_{2}\end{array}\right]$ and $A_{\tilde{\xi}}=\left[\begin{array}{ccc}0 & 0 & 0 \\ -k_{1} & -k_{2} & -1\end{array}\right]$.

Solving (22), we have

$e(t)=e^{A_{e} t} e(0)+\int_{0}^{t} e^{A_{e}(t-\tau)} A_{\tilde{\xi}} \tilde{\xi}(\tau) d \tau$.

According to (22) and Theorem 1, one has

$\left[A_{\tilde{\xi}} \tilde{\xi}(\tau)\right]_{i=1}=0$

$\left|\left[A_{\tilde{\xi}} \tilde{\xi}(\tau)\right]_{2}\right| \leq k_{\text {sum }} \sigma=\gamma \quad$ for all $t \geq T_{1}$

where $k_{\text {sum }}=1+k_{1}+k_{2}$. Let $\varphi(t)=\int_{0}^{t} e^{A_{e}(t-\tau)} A_{\tilde{\xi}} \tilde{\xi}(\tau) d \tau$. Define $\Psi=\left[\begin{array}{ll}0 & \gamma\end{array}\right]^{T}$. It follows that

$\left|\varphi_{i}(t)\right| \leq\left|\left(A_{e}^{-1} \Psi\right)_{i}\right|+\left|\left(A_{e}^{-1} e^{A_{e} t} \Psi\right)_{i}\right|$.

Since $A_{e}^{-1}=\left[\begin{array}{cc}-\frac{k_{2}}{k_{1}} & -\frac{1}{k_{1}} \\ 1 & 0\end{array}\right]=\left[\begin{array}{cc}-\frac{2}{\omega_{c}} & -\frac{1}{\omega_{c}^{2}} \\ 1 & 0\end{array}\right]$,we have

$\left|\left(A_{e}^{-1} \Psi\right)_{1}\right|=\frac{\gamma}{\omega_{c}^{2}}$

$\left|\left(A_{e}^{-1} \Psi\right)_{2}\right|=0$.

Since $A_{e}$ is Hurwitz, there exists a finite time $T_{2}>0$ such that

$\left|\left[e^{A_{e} t}\right]_{i j}\right| \leq \frac{1}{\omega_{c}^{3}}$

for all $t \geq T_{2}, i, j=1,2$. Note that $T_{2}$ depends on $A_{e}$. Let $e^{A_{e} t}=$ $\left[\begin{array}{ll}o_{11} & o_{12} \\ o_{21} & o_{22}\end{array}\right]$ and $e_{\text {sum }}(0)=\left|e_{1}(0)\right|+\left|e_{2}(0)\right|$. It follows that

$\left|\left[e^{A_{e} t} e(0)\right]_{i}\right| \leq \frac{e_{\text {sum }}(0)}{\omega_{c}^{3}}$

for all $t \geq T_{2}, i=1,2$. Let $T_{3}=\max \left\{T_{1}, T_{2}\right\}$. We have

$\left|\left(e^{A_{e} t} \Psi\right)_{i}\right| \leq \frac{\gamma}{\omega_{c}^{3}}$

for all $t \geq T_{3}, i=1,2$, and

$\left|\left(A_{e}^{-1} e^{A_{e} t} \Psi\right)_{i}\right| \leq\left\{\begin{array}{l}\left.\frac{1+2 \omega_{c}}{\omega_{c}^{2}} \frac{\gamma}{\omega_{c}^{3}}\right|_{i=1} \\ \left.\frac{\gamma}{\omega_{c}^{3}}\right|_{i=2}\end{array}\right.$

for all $t \geq T_{3}$. From (25), (26) and (30), we obtain

$\left|\varphi_{i}(t)\right| \leq\left\{\begin{array}{l}\frac{\gamma}{\omega_{c}^{2}}+\left.\frac{1+2 \omega_{c}}{\omega_{c}^{2}} \frac{\gamma}{\omega_{c}^{3}}\right|_{i=1} \\ \left.\frac{\gamma}{\omega_{c}^{3}}\right|_{i=2}\end{array}\right.$

for all $t \geq T_{3}$. From (23), one has

$\left|e_{i}(t)\right| \leq\left|\left[e^{A_{e} t} e(0)\right]_{i}\right|+\left|\varphi_{i}(t)\right|$.

According to (28), (31)-(32), we have the equations given in Box I).

It has been shown above that, with plant dynamics largely unknown, the tracking error and its derivative are bounded and their upper bounds monotonously decrease with the observer and controller bandwidths. With the convergence of ESO and ADRC established, the simulation test results are presented below.

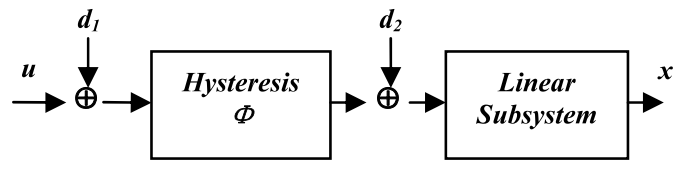

Fig. 1. Semi linear hysteretic system of (34).

\section{Simulation tests}

In this section, a typical hysteretic system is investigated to test the effectiveness of ADRC. Consider the following system:

$a_{n} x^{(n)}+a_{n-1} x^{(n-1)}+\cdots+a_{0} x=\Phi\left(u+d_{1}\right)+d_{2}$

where $d_{1}$ and $d_{2}$ are unknown disturbances. The system is shown in Fig. 1, representing the semi linear system (34).

The proposed ADRC is applied to the above semi linear system to demonstrate the capability to compensate for hysteresis. The hysteresis operator $\Phi\left(u+d_{1}\right)$ in the model is a unit normalized $\left(\alpha_{0}=1, \beta_{0}=-1\right)$ Preisach operator constructed from system response data. The operator is pre scaled and post scaled according to the $u(t)$ input measure dimension in the model. The simulation is normalized within a range of reference $x_{r}= \pm 1$ ref unit.

Fig. 2 represents the response of output versus input for the hysteresis data used for this simulation. The figure vividly illustrates major and minor hysteretic trajectories for increasing and decreasing input. One can readily see the response is not symmetric about the linear unit gain slope and introduces a very significant $(>50 \%)$ offset delay during both positive and negative input transitions. This hysteresis response is not only asymmetric about the unit slope, it is observed not even complementary in positive versus negative transitions. The example is purposefully extreme to best compare the commonly applied "inverse hysteresis model" compensation with the model independent ADRC/ESO compensation.

The hysteresis response plot is not continuous (readily observable) as it is interpolated from discrete data points ( 21 by 21). Finer resolution can be established at the expense of response speed when using the inverse model as part of the control in real time. This latter fact illustrates a major advantage of the ADRC/ESO, as it does not require the use of an inverse model of the hysteresis in order to compensate, thus avoiding this significant computation time penalty.

The linear subsystem used for this demonstration is a well understood 3rd order system comprised of a single integrator pole at the origin and two repeated negative real poles at $\omega_{n}=$ $1000 \mathrm{rad} / \mathrm{s}$ and with DC gain $b=\omega_{n}{ }^{2}$. This linear system was chosen as it is unstable, but may easily be made stable and well controlled using several strategies. The strategy chosen here assumes a full state feedback controller setting dominant closed loop negative complex poles at $10 \mathrm{k} \mathrm{rad} / \mathrm{s}$ with damping at 0.9 , plus one negative real pole at $100 \mathrm{k} \mathrm{rad} / \mathrm{s}$. The choice is arbitrary and illustrative rather than optimized to any cost function, showing a preference for transient response speed and accuracy over control energy cost.

Fig. 3 is a plot of the test reference signal used for this demonstration, having a peak value of 1 unit, not including any of the disturbances, offsets, noise, etc. The test reference signal is constituted of a trapezoid with $2 \mathrm{~ms}$ rise and fall times approximating the natural frequency, $\omega_{n}$, of the linear subsystem and an $8 \mathrm{~ms}$ extended quiescent period during which the disturbances and feedback noise will be introduced. The trapezoid is followed by $11 / 2$ sinusoid cycles at the natural frequency, $\omega_{n}=1000 \mathrm{rad} / \mathrm{s}$, of the linear subsystem. It is necessary here to note the test reference and disturbances are chosen near the natural dynamic limits of the system, many authors cite results of tests much lower in the system's dynamic spectrum, which may be easier to control accurately. 


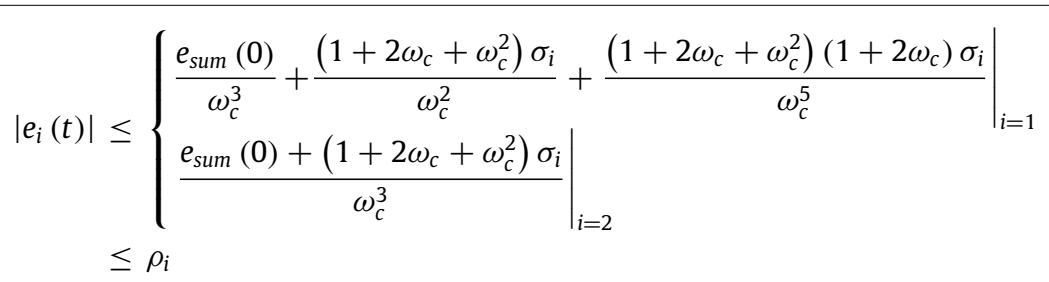

for $t \geq T_{3}, i=1,2$, where

$$
\rho_{i}=\max \left\{\frac{e_{\text {sum }}(0)}{\omega_{c}^{3}}+\frac{\left(1+2 \omega_{c}+\omega_{c}^{2}\right) \sigma_{i}}{\omega_{c}^{2}}+\frac{\left(1+2 \omega_{c}+\omega_{c}^{2}\right)\left(1+2 \omega_{c}\right) \sigma_{i}}{\omega_{c}^{5}}, \frac{e_{\text {sum }}(0)+\left(1+2 \omega_{c}+\omega_{c}^{2}\right) \sigma_{i}}{\omega_{c}^{3}}\right\} .
$$

Box I.

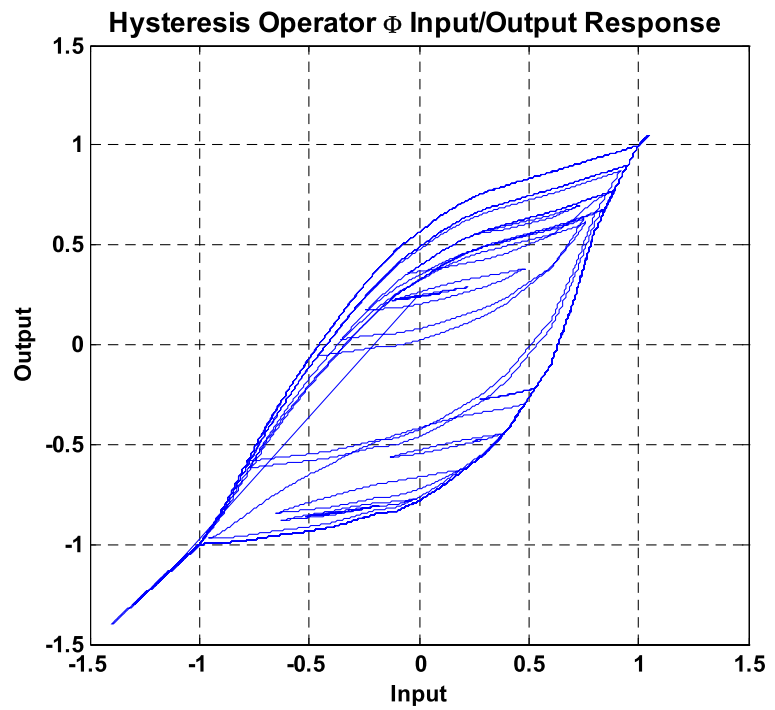

Fig. 2. Normalized hysteresis operator response.

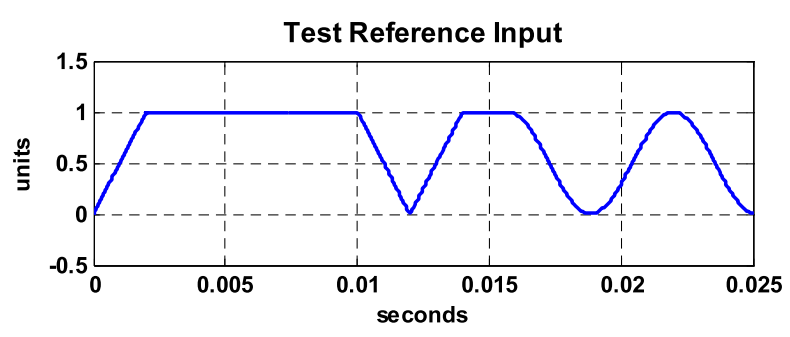

Fig. 3. The reference signal.

Fig. 4 shows the timing and magnitude of the control and load disturbances, $d_{1}$ and $d_{2}$ along with the measurement feedback noise, $n_{f}$. Disturbance $d_{1}$ is 0.5 unit pulse which multiplies the value of the control and then subtracts from the control signal between 4.7 and $6.3 \mathrm{~ms}$. Disturbance $d_{2}$ is 0.5 unit pulse which multiplies the value of the hysteresis output and then adds to the hysteresis signal between 7.8-9.4 ms. Measurement feedback noise $n_{f}$ is 0.1 unit peak $0.4 \mathrm{~ms}$ duration sinusoid repeated every $4 \mathrm{~ms}$ which is added to the measured output feedback signal. The noise sinusoid frequency varies randomly between 10 and $100 \mathrm{k} \mathrm{rad} / \mathrm{s}$.

Fig. 5 indicates the location where disturbance and noise are introduced into the system during simulation.

\subsection{Comparison of four scenarios}

Scenario \#1: The linear subsystem without hysteresis, controlled using a full state feedback controller designed as described

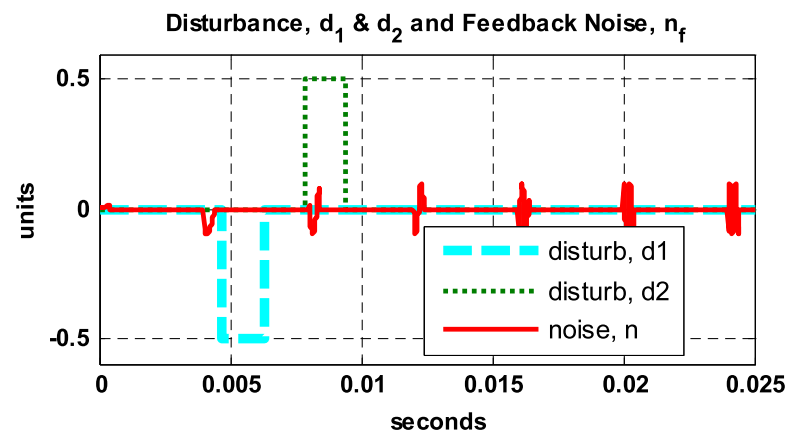

Fig. 4. The control output, $d_{1}$, and hysteresis output, $d_{2}$ disturbance signals, along with measurement feedback noise, $n_{f}$, signal.

previously in this section, and including control disturbance, $d_{1}$, and feedback noise, $n_{f}$, injected as described.

Scenario \#2: The linear subsystem with the hysteresis defined and illustrated in Fig. 2 placed in series as in the semi linear system of (34) and shown in Fig. 5 including both disturbances and noise as described. The controller is the same full state feedback controller as used in scenario \#1, without hysteresis compensation.

Scenario \#3: The linear subsystem and series hysteresis as in scenario \#2 including both disturbances and noise as described. The controller is the same full state feedback controller as used in scenario \#1 and \#2, however, with the addition of inverse hysteresis operator compensation, $\Phi^{-1}(u(t))$. The addition of this inverse operator, $\Phi^{-1}(u(t))$, as part of the controller follows the most common practice for hysteresis control.

Scenario \#4: The linear subsystem and series hysteresis as in scenario \#2 and \#3 including both disturbances and noise as described. The design and tuning of the ADRC/ESO are described next.

\subsection{Design and tuning of the ADRC/ESO}

The primary design parameter for the ADRC is the control signal coefficient $b$. In the scenario \#4, it is known from the linear subsystem DC gain $b=\omega_{n}^{2}$. The complete implementation requires tuning of the observer and controller bandwidth $\omega_{o}$ and $\omega_{c}$. One may optimize tuning by choosing a cost function and utilizing some iterative search algorithm, or one may quickly arrive at a usable solution via heuristic. A tuning heuristic is applied in this simulation whereby the ESO bandwidth, $\omega_{0}$, is set to $100 \mathrm{X}$ the linear system usable bandwidth $\omega_{0}=100 \omega_{n}$ and the controller bandwidth $\omega_{c}=\omega_{o} / 3$. The controller is tuned incrementally by first doubling or halving the observer bandwidth to approach the design goals, once near to the desired response one can reduce the increments. In this simulation scenario the tuning process was terminated arbitrarily with $\omega_{0}=300 \omega_{n}$ and $\omega_{c}=\omega_{0} / 3=$ $100 \omega_{n}$, again showing the same preference for response speed and 


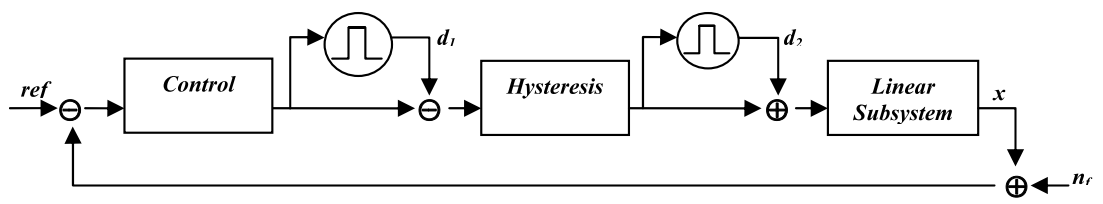

Fig. 5. Insertion points for control output, $d_{1}$, and hysteresis output, $d_{2}$ disturbance signals, along with measurement feedback noise, $n_{f}$, in the simulation.

accuracy over control energy cost as was done for the linear full state feedback control.

No inverse hysteresis operator is required for this ADRC/ESO control scenario, the hysteresis is treated as part of the estimated nonlinear function $f(\cdot)$, as are other unknown disturbance to the desired response, and compensated directly. The ADRC/ESO design thus requires minutes versus the hours/days/weeks required to experimentally measure the hysteresis inverse response data.

\subsection{Performance of the ADRC/ESO versus inverse hysteresis compen- sation using $\Phi^{-1}(u(t))$}

The comparison of the four different scenario's response to the test reference signal including disturbances and noise are remarkable.

Fig. 6a illustrates the response of the scenario \#1 linear subsystem with linear full state feedback controller. The phase lag for the closed loop system measured at $22 \mathrm{~ms}$ is $0.2 \mathrm{~ms}=11.5^{\circ}$ contributing to a tracking error of $\sim 9 \%$. One can observe the full state feedback control responds to the disturbance $d_{1}$ quite well, without error. However, the control does not well compensate the measurement feedback noise, $n_{f}$, which results in $\sim 8 \%$ error at $10 \mathrm{k} \mathrm{rad} / \mathrm{s}$ noise frequency, rolling off to $\sim 2 \%$, as expected, as the noise frequency rises toward $100 \mathrm{k} \mathrm{rad} / \mathrm{s}$. The average absolute value of error over the $25 \mathrm{~ms}$ duration of the simulation is 0.0494 with a corresponding average absolute value of control effort of $3.2623 \times 10^{4}(n=250,001$ samples in $25 \mathrm{~ms})$.

$A v g \cdot A b s \cdot$ Error $=\left(\sum_{m=1}^{n} \mid\right.$ error $\left._{m} \mid\right) / n$.

Fig. 6b illustrates the response of the scenario \#2 semi linear system with hysteresis, without compensation. The controller is the same linear full state feedback controller as scenario \#1. The lack of hysteresis compensation renders the standard linear full state feedback controller unacceptable. The phase lag for the closed loop system measured at $22 \mathrm{~ms}$ is unchanged, $0.2 \mathrm{~ms}=11.5^{\circ}$ contributing to a tracking error of $\sim 9 \%$, so that the remaining hysteresis offset error is approximately $40 \%$. It must be noted the recovery response from the disturbance, $d_{1}$, at $6.3 \mathrm{~ms}$ is coincidental, in that the hysteresis offset and disturbance offset almost cancel each other, this would not be true had the disturbance been more or less. The linear controller does compensate for disturbance, $d_{2}$, which is not affected by the hysteresis. The control reacts to measurement feedback noise, $n_{f}$, similar to scenario \#1, rolling off as expected as the noise frequency rises toward $100 \mathrm{k} \mathrm{rad} / \mathrm{s}$. The uncompensated hysteresis has increased the average absolute value of error over the $25 \mathrm{~ms}$ duration of the simulation five-fold, to 0.1906 , while also requiring an additional 50 fold increase in average absolute value of control effort to $1.8246 \times 10^{6}$.

Fig. 6c illustrates scenario \#3, the semi linear system with hysteresis, now with inverse hysteresis compensation incorporated in the controller. The positive effect of the compensation is as expected from the results reported by many authors who have adopted this now standard linearization methodology. The closed loop response is very nearly the same as the scenario \# 1 linear system without hysteresis. The phase lag for the closed loop system
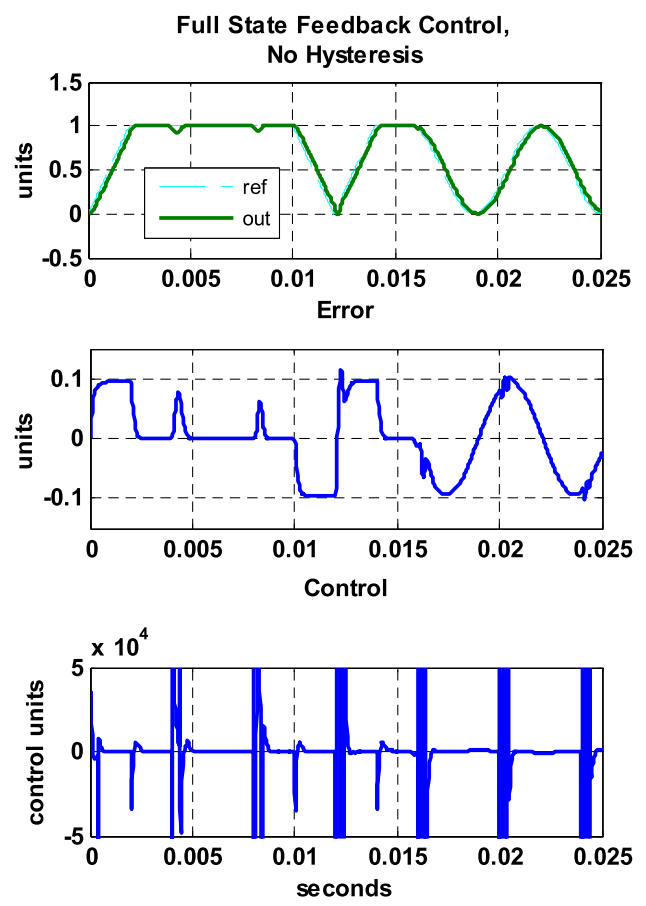

Fig. 6a. Scenario \#1 result: phase lag error $\sim 9 \%, A v g \cdot$ Abs $\cdot$ Error $=0.0494$ Avg $\cdot$ Abs $\cdot$ Control $=3.2623 \times 10^{4}$.
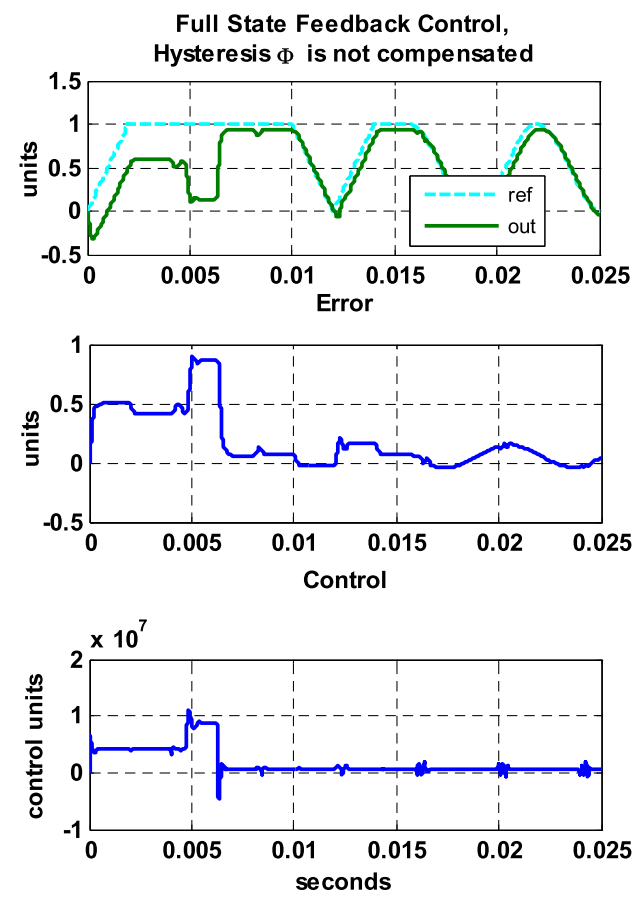

Fig. 6b. Scenario \#2 result: hysteresis error $\sim 40 \%, A v g \cdot A b s \cdot$ Error $=0.1906$, Avg $\cdot$ Abs $\cdot$ Control $=1.8246 \times 10^{6}$

measured at $22 \mathrm{~ms}$ is unchanged, $0.2 \mathrm{~ms}=11.5^{\circ}$ contributing to a tracking error of $\sim 9 \%$, so that the remaining hysteresis offset error 

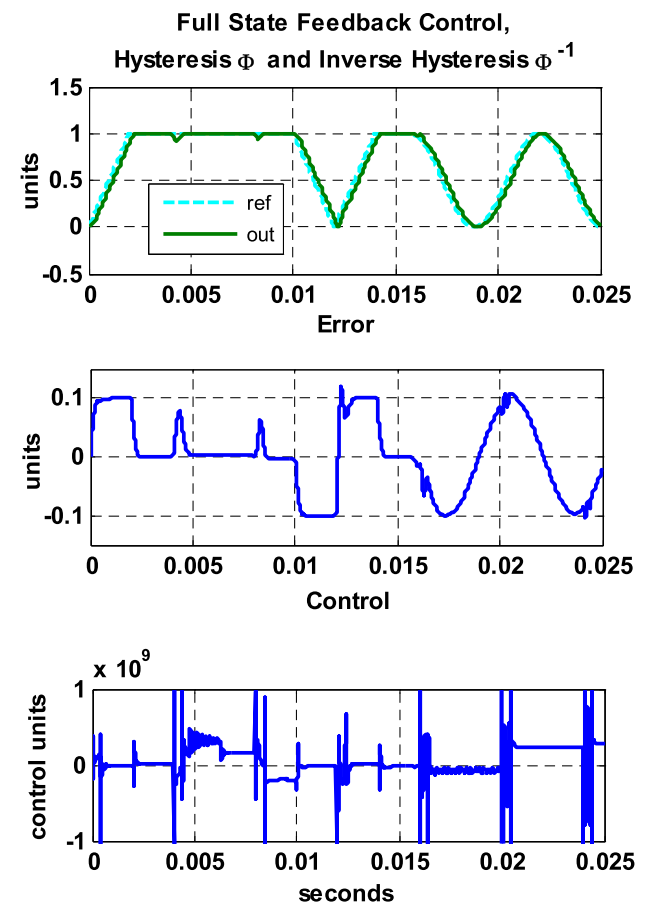

Fig. 6c. Scenario \#3 result: phase + hysteresis error $\sim 10 \%, A v g \cdot A b s \cdot$ Error $=$ 0.0491, Avg $\cdot$ Abs $\cdot$ Control $=1.5392 \times 10^{8}$.

is only $\sim 1 \%$, similar to the results reported by many authors. The hysteresis compensated full state feedback controller performs the job of controlling disturbance $d_{1}$ and $d_{2}$ without error. The control reacts to measurement feedback noise, $n_{f}$, almost exactly as scenario \#1 without hysteresis, rolling off as expected as the noise frequency rises toward $100 \mathrm{k} \mathrm{rad} / \mathrm{s}$. The hysteresis compensated controller has matched the scenario \#1 "no hysteresis" linear system average absolute value of error over the $25 \mathrm{~ms}$ duration of the simulation, at 0.0491 , however requiring a significant 5000 fold increase in average absolute value of control effort to $1.5392 \times 10^{8}$. This then sets the standard to which the ADRC/ESO must be compared.

Fig. 6d illustrates the response of the ADRC/ESO design scenario \#4. The reader is reminded the ADRC/ESO has no separate inverse hysteresis compensator, the hysteresis error is estimated as part of the unknown disturbance. The character of the ADRC/ESO response is different in almost every way. The most obvious difference is the response to disturbance, $d_{1}$, which is then further influenced by the hysteresis. The common linear controller with inverse hysteresis compensation, scenario \#3, rendered the closed loop system response error free when disturbed, conversely, the faster responding ADRC/ESO controller continually overshoots the reference, leading to an oscillatory error $\pm 3.4 \%$ while this disturbance, $d_{1}$, is present. The reaction to disturbance $d_{2}$, not affected by hysteresis, is much less pronounced. The ADRC/ESO response to measurement feedback noise, $n_{f}$, is also more pronounced at exactly $\pm 10 \%$ and not diminished by the frequency, the ADRC/ESO responding instantaneously to the perceived change in reference input command. This active response, normally a strength, but in this regard a limitation even when bounded, must be addressed proactively when applying the ADRC/ESO.

The ability of ADRC/ESO to cancel the effect of linear system phase lag and nonlinear hysteresis is remarkable. The closed loop phase lag measured at $22 \mathrm{~ms}$ is now only $2.3^{\circ}$ and the total error due to phase lag and hysteresis offset is reduced to $\sim 1.5 \%$, about $1 / 6$ that of the inverse compensated controller. The ADRC/ESO controller average absolute value of error over the $25 \mathrm{~ms}$ duration of the simulation, at 0.0143 , is $70 \%$ less than the linear system with
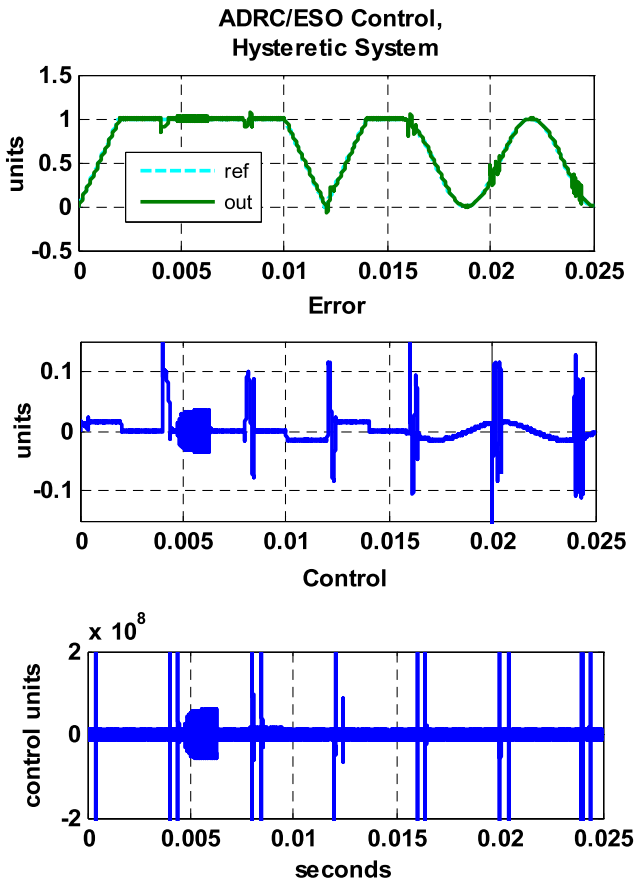

Fig. 6d. Scenario \#4 result: phase + hysteresis error $\sim 1.5 \%, A v g \cdot A b s \cdot$ Error $=$ 0.0143, Avg $\cdot$ Abs $\cdot$ Control $=1.6570 \times 10^{7}$.

no hysteresis, while requiring an average absolute value of control effort of $1.6570 \times 10^{7}$, only $11 \%$ of the control energy required by the common inverse hysteresis compensated control.

\section{Concluding remarks}

In this paper, the novel concept ADRC is successfully applied for hysteresis compensation whereby hysteresis is treated as a disturbance to the desired linear behavior and rejected. Simulation test comparisons on hysteresis dominated systems, which have immediate and significant research interest, demonstrate the effectiveness of this new method. This disturbance rejection strategy achieves superior results and requires minimal computational resource compared to model based inverse hysteresis controllers, thus it may be implemented at less cost and/or higher response speed. The achievement of this performance without resorting to the complexity of mathematic modeling is a significant advance. The stability analyses solidify the theoretical foundation of the proposed approach and provide much insight for the users, and the rationale for the success of simulation tests.

\section{Acknowledgment}

The authors would like to thank Pavel Krejci for his graciousness enabling access to his rare treatise, without which this work would not have been possible.

\section{References}

[1] Visitin A. Differential models of hysteresis. Berlin: Springer-Verlag; 1994.

[2] Tao G, Kokotovic PV. Adaptive control of plants with unknown hysteresis. IEEE Transactions on Automatic Control 1995;40:200-12.

[3] Krejci P. Hysteresis, convexity and dissipation in hyperbolic equations. GAKUTO International Series. Mathematical Sciences and Applications $1996 ; 8$.

[4] Brokate M, Sprekels J. Hysteresis and phase transitions. Appl. math. sci., Vol. 121. Springer-Verlag; 1996. 
[5] Mittal S, Menq $\mathrm{CH}$. Hysteresis compensation in electromagnetic actuators through preisach model inversion. IEEE/ASME Transactions on Mechatronics 2000;5(4):394.

[6] Mayergoyz I. Mathematical models of hysteresis and their applications. Academic Press; 2003.

[7] Logemann H, Ryan EP. Systems with hysteresis in the feedback loop: existence, regularity and asymptotic behaviour of solutions. ESAIM: Control, Optimisation and Calculus of Variations 2003;9:169-96.

[8] Tan X, Baras J. Modeling and control of hysteresis in magnetostrictive actuators. Automatica 2004;40:1469-80.

[9] Iyer R, Tan X. Control of hysteretic systems through inverse compensation. IEEE Control Systems Magazine 2009;29(1):93-9.

[10] Yi J, Chang S, Shen Y. Disturbance-observer-based hysteresis compensation for piezoelectric actuators. IEEE/ASME Transactions on Mechatronics 2009;14(4): 456-64.

[11] Al Janaideh M, Feng Y, Rajheja S, Su CY, Rabbath C. Hysteresis compensation for smart actuators using inverse generalized Prandtl-Ishlinskii model. Proceedings of the American Control Conference 2009;307-12.

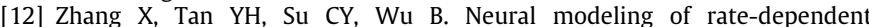
and asymmetric hysteresis in ultrasonic motors. Proceedings of the IEEE Conference on Decision and Control 2009;727-32.

[13] Ruderman M, Bertram T. Discrete dynamic preisach model for robust inverse control of hysteresis systems. Proceedings of the IEEE Conference on Decision and Control 2010;3463-8.

[14] Juhasz J, Maas J, Borovac B. Parameter identification and hysteresis compensation of embedded piezoelectric stack actuators. Mechatronics 2011;21: 329-38.
[15] Tan YZ, Pang CK, Hong F, Won S, Lee TH. Hysteresis compensation of piezoelectric actuators in dual-stage hard disk drives. Proceedings of the Asian Control Conference 2011;1024-9.

[16] Gao Z. Scaling and parameterization based controller tuning. Proceedings of the American Control Conference 2003;4989-96.

[17] Gao Z. Active disturbance rejection control: a paradigm shift in feedback control system design. Proceedings of the American Control Conference 2006; 2399-405.

[18] Johnson C. Accommodation of external disturbances in linear regulator and servomechanism problems. IEEE Transactions on Automatic Control 1971;AC16(6):635-44.

[19] Chen J, Patton RJ, Zhang H. Design of unknown input observers and robust fault detection filters. International Journal of Control 1995;63(1):85-105.

[20] Schrijver E, van Dijk J. Disturbance observers for rigid mechanical systems: equivalence, stability, and design. ASME Journal of Dynamics Systems, Measurement, and Control 2002;124(4):539-48.

[21] Yang K, Choi Y, Chung W. On the tracking performance improvement of optical disk drive servo systems using error-based disturbance observer. IEEE Transactions on Industrial Electronics 2005;52(1):270-9.

[22] Kwon S, Chung WK. Combined synthesis of state estimator and perturbation observer. ASME Journal of Dynamic Systems, Measurement, and Control 2003 125:19-26.

[23] Gao Z, Huang Y, Han J. An alternative paradigm for control system design. Proceedings of IEEE conference on Decision and Control 2001;4578-85.

[24] Zheng Q, Chen Z, Gao Z. A practical approach to disturbance decoupling control. Control Engineering Practice 2009;17(9):1016-25. 